\title{
Green's functions for 2.5D elastodynamic problems in a free solid layer formation
}

\author{
António Tadeu *, Julieta António \\ University of Coimbra, Department of Civil Engineering, 3030-290 Coimbra, Portugal
}

Received 2 May 2001; received in revised form 1 October 2001; accepted 1 October 2001

\begin{abstract}
This work presents analytical Green's functions for the steady state response of a homogeneous three-dimensional free solid layer formation (slab) subjected to a spatially sinusoidal harmonic line load, polarized along the horizontal, vertical and $z$ directions. The equations presented here are not only themselves very interesting but are also useful for formulating three-dimensional elastodynamic problems in a slab-type formation, using integral transform methods and/or boundary elements. The final expressions are validated by comparing them with the results obtained by using the Boundary Element Method solution, for which both free surfaces of the slab are discretized with boundary elements. (c) 2002 Elsevier Science Ltd. All rights reserved.
\end{abstract}

Keywords: Green's functions; Free solid layer formation; Analytical solutions

\section{Introduction}

In an earlier work, the authors provided the Green's functions for computing the wavefield produced by a harmonic point load buried in a half-space [1]. These functions, relating the stresses or displacements at some location in the half-space domain, caused by a dynamic source placed elsewhere in the medium, were proven to be both very useful themselves and also of great interest to techniques requiring the full discretization of the halfspace surface, such as the Boundary Element Method (BEM).

The present paper expands this earlier work. It presents the Green's functions for calculating the wavefield produced in a homogeneous three-dimensional free solid layer formation (slab), subjected to a spatially sinusoidal harmonic line load, polarized along the horizontal, vertical and $z$ directions, as in Fig. 1(b). The technique starts out knowing the solid displacement potentials employed to evaluate the Green's functions for a harmonic (steady state) line load with a sinusoidally varying amplitude in the third dimension, in an

* Corresponding author. Tel.: +351-239-797-201; fax: +351-239797-190.

E-mail address: tadeu@dec.uc.pt (A. Tadeu). unbounded medium [2]. All displacement potentials are written as a superposition of plane waves following the approach used first by Lamb [3] for the two-dimensional case, and then by Bouchon [4] and Kim et al. [5] to compute the three space dimension field by means of a discrete wave number representation. The Green's functions for the slab formation are then derived, ascribing null stresses to both surfaces of the slab. This procedure involves writing the final Green's functions as the sum of the Green's function for a full-space with surface terms, produced by the slab's two free surfaces, using a technique similar to that described by Kawase [6]. The Green's functions presented here for a spatially sinusoidal harmonic line (steady state) load in a free layer solid formation, are often referred to in the literature as the $2.5 \mathrm{D}$ problem. The equations are not only intrinsically very important, they are also very useful for formulating 3D elastodynamic problems, such as those involving the discretization of both free surfaces, via a Boundary Element Method.

This paper has three parts. First, there is a brief description showing how the Green's functions for a sinusoidal line load, applied in an unbounded solid formation along the $x, y$ and $z$ directions, can be calculated as a continuous superposition of plane waves. Then, the Green's functions for a free solid layer formation are established, using the required boundary conditions at 
(a)

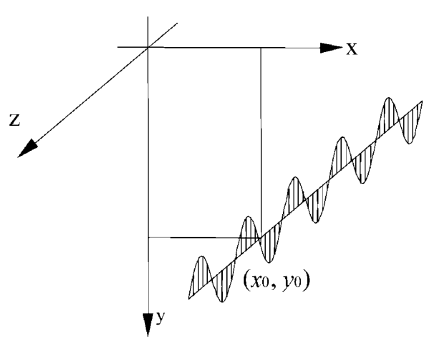

(b)

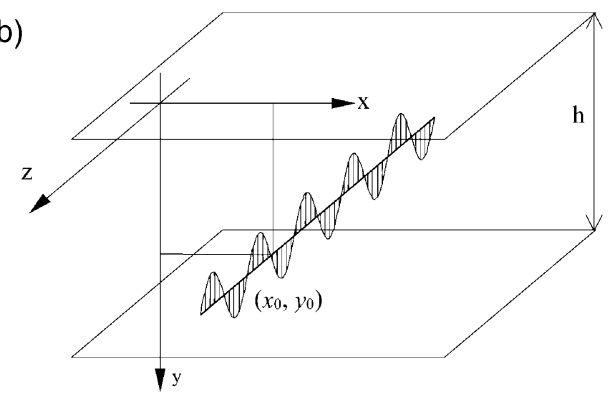

Fig. 1. Geometry of the problem: (a) Full-space; (b) Slab.

both surfaces. Finally, the full set of expressions is compared with those provided by the BEM, for which a full discretization of both free layer surfaces is required.

\section{Green's functions in an unbounded medium}

An unbounded, homogeneous space is perturbed by a spatially varying line load of the form $p\left(x_{0}, y_{0}, t\right)=\delta(x-$ $\left.x_{0}\right) \delta\left(y-y_{0}\right) \mathrm{e}^{i\left(\omega t-k_{z} z\right)}$ applied at $\left(x_{0}, y_{0}\right)$, acting in one of the three coordinate directions. In this equation, $\delta\left(x-x_{0}\right)$ and $\delta\left(y-y_{0}\right)$ are Dirac-delta functions, $\omega$ is the frequency of the load and $k_{z}$ is the wavenumber in $z$ (see Fig. 1(a)). The solution for this load can be obtained by applying a spatial Fourier transform in the $z$ direction to the Helmholtz equations for a point load (see, e.g., [7]). The $z$ transformed equations are then

$$
\begin{array}{r}
\left(\frac{\partial^{2} \hat{A}_{p}}{\partial x^{2}}+\frac{\partial^{2} \hat{A}_{p}}{\partial y^{2}}+k_{\alpha}^{2} \hat{A}_{p}\right)=\frac{-i H_{0}^{(2)}\left(-i k_{z} r\right)}{4 \rho \alpha^{2}} \\
\left(\frac{\partial^{2} \hat{A}_{s}}{\partial x^{2}}+\frac{\partial^{2} \hat{A}_{s}}{\partial y^{2}}+k_{\beta}^{2} \hat{A}_{s}\right)=\frac{-i H_{0}^{(2)}\left(-i k_{z} r\right)}{4 \rho \beta^{2}}
\end{array}
$$

where $k_{\alpha}=\sqrt{k_{p}^{2}-k_{z}^{2}} \quad$ with $\quad\left(\operatorname{Im}\left(k_{\alpha}\right) \leq 0\right) \quad$ and $\quad k_{p}=\omega / \alpha$, $k_{\beta}=\sqrt{k_{s}^{2}-k_{z}^{2}}$ with $\left(\operatorname{Im}\left(k_{\beta}\right) \leq 0\right)$ and $k_{s}=\omega / \beta, \alpha=\sqrt{(\lambda+2 \mu) / \rho}$ and $\beta=\sqrt{\mu / \rho}$ are the velocities for $P$ (pressure) waves and $S$ (shear) waves, respectively, $\lambda$ and $\mu$ are the Lamé constants, $\rho$ is the mass density, $\hat{A}_{p}\left(x, y, k_{z}, \omega\right)$ and $\hat{A}_{s}\left(x, y, k_{z}, \omega\right)$ are the Fourier transforms of the two potentials $A_{p}(x, y, z, \omega)$ and $A_{s}(x, y, z, \omega)$ for the irrotational and equivoluminal parts of the displacement vector, $H_{n}^{(2)}()$ are Hankel functions of the second kind and $n$th order, $r=\sqrt{\left(x-x_{0}\right)^{2}+\left(y-y_{0}\right)^{2}}$ and $i=\sqrt{-1}$. From equilibrium conditions it is possible to obtain $\hat{A}_{p}$ and $\hat{A}_{s}$,

$$
\begin{aligned}
& \hat{A}_{p}=\frac{i}{4 \rho \omega^{2}}\left[H_{0}^{(2)}\left(k_{\alpha} r\right)-H_{0}^{(2)}\left(-i k_{z} r\right)\right] \\
& \hat{A}_{s}=\frac{i}{4 \rho \omega^{2}}\left[H_{0}^{(2)}\left(k_{\beta} r\right)-H_{0}^{(2)}\left(-i k_{z} r\right)\right]
\end{aligned}
$$

The displacements $G_{i j}$ in direction $i$, due to a load applied in direction $j$, can then be obtained from the relation
$G_{i j}=\frac{\partial^{2}\left(\hat{A}_{p}-\hat{A}_{s}\right)}{\partial x_{i} \partial x_{j}}+\delta_{i j} \bar{\nabla}^{2} \hat{A}_{s}$

in which $\delta_{i j}$ is the Kronecker delta, $x_{j}=x, y, z$ for $j=1,2$, 3 , and $(\partial / \partial z)=-i k_{z}$. We may observe that

$$
\begin{aligned}
& \hat{A}_{p}-\hat{A}_{s}=\frac{1}{4 i \rho \omega^{2}}\left[H_{0}^{(2)}\left(k_{\beta} r\right)-H_{0}^{(2)}\left(k_{\alpha} r\right)\right] \\
& \bar{\nabla}^{2} \hat{A}_{s}=\frac{1}{4 i \rho \beta^{2}} H_{0}^{(2)}\left(k_{\beta} r\right)
\end{aligned}
$$

Tadeu and Kausel [2] gives a full set of Green's functions, expressions for the strains and stresses, which fully agree with the solution for moving loads given earlier by Pedersen et al. [8] and Papageorgiou et al. [9].

In order to obtain the Green's functions that apply to the slab formation, the above equations must be expressed as a continuous superposition of homogeneous and inhomogeneous plane waves when the load acts in the direction $x, y$ and $z$.

\section{Green's functions in a free solid layer formation}

\subsection{Load acting in the direction of the $x$-axis}

The displacement potentials generated by a spatially sinusoidal harmonic line load along the $z$ direction, applied at the point $\left(x_{0}, y_{0}\right)$ in the $x$ direction, are given by the expressions,

$$
\begin{aligned}
& \phi^{x}=\frac{1}{4 \pi \rho \omega^{2}} \int_{-\infty}^{+\infty}\left(\frac{k}{v} \mathrm{e}^{-i v\left|y-y_{0}\right|}\right) \mathrm{e}^{-i k\left(x-x_{0}\right)} \mathrm{d} k \\
& \psi_{x}^{x}=0 \\
& \psi_{y}^{x}=\frac{i\left(-i k_{z}\right)}{4 \pi \rho \omega^{2}} \int_{-\infty}^{+\infty}\left(\frac{\mathrm{e}^{-i \gamma\left|y-y_{0}\right|}}{\gamma}\right) \mathrm{e}^{-i k\left(x-x_{0}\right)} \mathrm{d} k \\
& \psi_{z}^{x}=\frac{-\operatorname{sgn}\left(y-y_{0}\right)}{4 \pi \rho \omega^{2}} \int_{-\infty}^{+\infty}\left(\mathrm{e}^{-i \gamma\left|y-y_{0}\right|}\right) \mathrm{e}^{-i k\left(x-x_{0}\right)} \mathrm{d} k
\end{aligned}
$$


where $v=\sqrt{k_{p}^{2}-k_{z}^{2}-k^{2}}$ with $(\operatorname{Im~ag}(v) \leq 0), \quad \gamma=\sqrt{k_{s}^{2}-k_{z}^{2}-k^{2}}$ with $(\operatorname{Im}(\gamma) \leq 0)$, and the integration relates to the horizontal wave number, $k$, along the $x$ direction.

These integrals can be obtained as a discrete summation, assuming the existence of an infinite number of such sources distributed along the $x$ direction, at equal intervals $L_{x}$. The above compressional and rotational potentials can then be written as

$$
\begin{aligned}
& \phi^{x}=E_{a} \sum_{n=-\infty}^{n=+\infty}\left(\frac{k_{n}}{v_{n}} E_{b}\right) E_{d} \\
& \psi_{x}^{x}=0 \\
& \psi_{y}^{x}=E_{a} k_{z} \sum_{n=-\infty}^{n=+\infty}\left(\frac{E_{c}}{\gamma_{n}}\right) E_{d} \\
& \psi_{z}^{x}=-\operatorname{sgn}\left(y-y_{0}\right) E_{a} \sum_{m=-\infty}^{n=+\infty}\left(E_{c}\right) E_{d}
\end{aligned}
$$

where

$$
\begin{aligned}
& E_{a}=\frac{1}{2 \rho \omega^{2} L_{x}} \\
& E_{b}=\mathrm{e}^{-i v_{n}\left|y-y_{0}\right|} \\
& E_{c}=\mathrm{e}^{-i \gamma_{n}\left|y-y_{0}\right|} \\
& E_{d}=\mathrm{e}^{-i k_{n}\left(x-x_{0}\right)} . \\
& v_{n}=\sqrt{k_{p}^{2}-k_{z}^{2}-k_{n}^{2}} \text { with }\left(\operatorname{Im}\left(v_{n}\right) \leq 0\right) \\
& \gamma_{n}=\sqrt{k_{s}^{2}-k_{z}^{2}-k_{n}^{2}} \text { with }\left(\operatorname{Im}\left(\gamma_{n}\right) \leq 0\right) \\
& k_{n}=\frac{2 \pi}{L_{x}} n
\end{aligned}
$$

which can be approximated by a finite sum of terms $(N)$.

The Green's functions for an unbounded medium can therefore be expressed in terms of the compressional and rotational potentials, $\phi^{x}, \psi_{x}^{x}, \psi_{y}^{x}$ and $\psi_{z}^{x}$, from which the following three components of displacement can be calculated,

$$
\begin{aligned}
& G_{x x}^{\mathrm{full}}=E_{a} \sum_{n=-N}^{n=+N}\left[\frac{-i k_{n}^{2}}{v_{n}} E_{b}+\left(-i \gamma_{n}-\frac{i k_{z}^{2}}{\gamma_{n}}\right) E_{c}\right] E_{d} \\
& G_{y x}^{\mathrm{full}}=E_{a} \sum_{n=-N}^{n=+N}\left[-i \operatorname{sgn}\left(y-y_{0}\right) k_{n} E_{b}+i \operatorname{sgn}\left(y-y_{0}\right) k_{n} E_{c}\right] E_{d} \\
& G_{z x}^{\mathrm{full}}=E_{a} \sum_{n=-N}^{n=+N}\left(\frac{-i k_{z} k_{n}}{v_{n}} E_{b}+\frac{i k_{z} k_{n}}{\gamma_{n}} E_{c}\right) E_{d}
\end{aligned}
$$

The Green's functions for a free solid layer formation (slab), with thickness $h$, can be expressed as the sum of the source terms equal to those in the full-space and the surface terms needed to satisfy the boundary conditions at the two free stress surfaces (null normal and tangential stresses). Both interfaces (top and bottom) generate surface terms which can be expressed in a form similar to that of the source term,

\subsection{Solid medium (top free surface)}

$$
\begin{aligned}
& \phi_{0}^{x-\text { top }}=E_{a} \sum_{n=-\infty}^{n=+\infty}\left(\frac{k_{n}}{v_{n}} E_{b 0} A_{n}^{x}\right) E_{d} \\
& \psi_{x 0}^{x-\text { top }}=0 \\
& \psi_{y 0}^{x-\text { top }}=E_{a} k_{z} \sum_{n=-\infty}^{n=+\infty}\left(\frac{E_{c 0}}{\gamma_{n}} B_{n}^{x}\right) E_{d} \\
& \psi_{z 0}^{x-\text { top }}=-E_{a} \sum_{n=-\infty}^{n=+\infty}\left(E_{c 0} C_{n}^{x}\right) E_{d}
\end{aligned}
$$

\subsection{Solid medium (bottom free surface)}

$$
\begin{aligned}
& \phi_{0}^{x \text { bottom }}=E_{a} \sum_{n=-\infty}^{n=+\infty}\left(\frac{k_{n}}{v_{n}} E_{b 0}^{b} D_{n}^{x}\right) E_{d} \\
& \psi_{x 0}^{x-\text { bottom }}=0 \\
& \psi_{y 0}^{x}{ }^{x-\text { bottom }}=E_{a} k_{z} \sum_{n=-\infty}^{n=+\infty}\left(\frac{E_{c 0}^{b}}{\gamma_{n}} E_{n}^{x}\right) E_{d} \\
& \psi_{z 0}^{x-\text { bottom }}=-E_{a} \sum_{n=-\infty}^{n=+\infty}\left(E_{c 0}^{b} F_{n}^{x}\right) E_{d}
\end{aligned}
$$

where, $E_{b 0}=\mathrm{e}^{-i v_{n} y}, E_{c 0}=\mathrm{e}^{-i \gamma_{n} y}, E_{b 0}^{b}=\mathrm{e}^{-i v_{n}|y-h|}, E_{c 0}^{b}=\mathrm{e}^{-i \gamma_{n}|y-h|}$, $A_{n}^{x}, B_{n}^{x}, C_{n}^{x}, D_{n}^{x}, E_{n}^{x}$ and $F_{n}^{x}$ are as yet unknown coefficients to be defined by imposing the appropriate boundary conditions, so that the field produced simultaneously by the source and the surface terms guarantees $\sigma_{y x}^{s}=0, \sigma_{y z}^{s}=0$ and $\sigma_{y y}^{s}=0$ at $y=0$ and at $y=h$.

The imposition of the sixth stated boundary conditions for each value of $n$ leads to a system of six equations in the six unknown constants. The derivation of the total system of equations is quite straightforward, but the details are rather unwieldy, and for this reason the final system of equations alone is presented here

$$
\left[a_{i j}^{x} i=1,6 ; j=1,6\right]\left[c_{i}^{x} i=1,6\right]=\left[b_{i}^{x} i=1,6\right]
$$

but the full derivation is described in Appendix B.

Once this system of equations has been solved, the amplitude of the surface potentials has been fully defined, and thus the displacements relative to the slab can be obtained. The final expressions for the Green's functions are then obtained from the sum of the source terms and the surface terms originated in the two free surfaces, which leads to the following expressions,

$G_{x x}^{\mathrm{slab}}=G_{x x}^{\mathrm{full}}+E_{a} \sum_{n=-N}^{n=+N}\left[A_{n}^{x} \frac{-i k_{n}^{2}}{v_{n}} E_{b 0}+\left(-i \gamma_{n} C_{n}^{x}\right.\right.$ 


$$
\begin{aligned}
& \left.\left.-\frac{i k_{z}^{2}}{\gamma_{n}} B_{n}^{x}\right) E_{c 0}\right] E_{d}+E_{a} \sum_{n=-N}^{n=+N}\left[D_{n}^{x} \frac{-i k_{n}^{2}}{v_{n}} E_{b 0}^{b}+\left(-i \gamma_{n} F_{n}^{x}\right.\right. \\
& \left.\left.-\frac{i k_{z}^{2}}{\gamma_{n}} E_{n}^{x}\right) E_{c 0}^{b}\right] E_{d} \\
& G_{y x}^{\text {slab }}=G_{y x}^{\mathrm{full}}+E_{a} \sum_{n=-N}^{n=+N}\left(-i k_{n} A_{n}^{x} E_{b 0}+i k_{n} C_{n}^{x} E_{c 0}\right) E_{d} \\
& +E_{a} \sum_{n=-N}^{n=+N}\left(i k_{n} D_{n}^{x} E_{b 0}^{0}-i k_{n} F_{n}^{x} E_{c 0}^{b}\right) E_{d} \\
& G_{z x}^{\mathrm{slab}}=G_{z x}^{\mathrm{full}}+E_{a} \sum_{n=-N}^{n=+N}\left(\frac{-i k_{z} k_{n}}{v_{n}} A_{n}^{x} E_{b 0}+\frac{i k_{z} k_{n}}{\gamma_{n}} B_{n}^{x} E_{c 0}\right) E_{d} \\
& +E_{a} \sum_{n=-N}\left(\frac{-i k_{z} k_{n}}{v_{n}} D_{n}^{x} E_{b 0}^{b}+\frac{i k_{z} k_{n}}{\gamma_{n}} E_{n}^{x} E_{c 0}^{b}\right) E_{d}
\end{aligned}
$$

The expressions for the Green's function for two-anda-half dimensional full space $G_{x x}^{\text {full }}, G_{y x}^{\text {full }}$ and $G_{z x}^{\text {full }}$ can be defined in explicit form, as listed in Appendix A [2]. The Green's functions for loads applied along the $y$ and $z$ directions can be computed following a similar procedure. The derivation of these solutions is then presented, but in condensed form.

\subsection{Load acting in the direction of the $y$-axis}

The discrete form of the displacement potentials, for a spatially sinusoidal harmonic line load along the $z$ direction, applied at the point $\left(x_{0}, y_{0}\right)$ in the $y$ direction, is now given by the expressions,

$$
\begin{aligned}
& \phi^{y}=E_{a} \sum_{n=-N}^{n=+N}\left[\operatorname{sgn}\left(y-y_{0}\right) E_{b}\right] E_{d} \\
& \psi_{x}^{y}=E_{a} k_{z} \sum_{n=-N}^{n=+N}\left(\frac{-E_{c}}{\gamma_{n}}\right) E_{d} \\
& \psi_{y}^{y}=0 \\
& \psi_{z}^{y}=E_{a} \sum_{n=-N}^{n=+N}\left(\frac{k_{n}}{\gamma_{n}} E_{c}\right) E_{d}
\end{aligned}
$$

The Green's functions for a two-and-a-half dimensional full space are thus,

$$
\begin{aligned}
& G_{x y}^{\mathrm{full}}=G_{y x}^{\mathrm{full}}=E_{a} \sum_{n=-N}^{n=+N}\left[-i \operatorname{sgn}\left(y-y_{0}\right) k_{n} E_{b}\right. \\
& \left.+i \operatorname{sgn}\left(y-y_{0}\right) k_{n} E_{c}\right] E_{d} \\
& G_{y y}^{\mathrm{full}}=E_{a} \sum_{n=-N}^{n=+N}\left[-i v_{n} E_{b}+\left(\frac{i v_{z n}^{2}}{\gamma_{n}}\right) E_{c}\right] E_{d} \\
& G_{z y}^{\mathrm{full}}=E_{a} \sum_{n=-N}^{n=+N}\left[-i \operatorname{sgn}\left(y-y_{0}\right) k_{z} E_{b}+i \operatorname{sgn}\left(y-y_{0}\right) k_{z} E_{c}\right] E_{d}
\end{aligned}
$$

The surface terms generated at the two free slab surfaces can be expressed in the form

\subsection{Solid medium (top free surface)}

$$
\begin{aligned}
& \phi_{0}^{y-\text { top }}=E_{a} \sum_{n=-N}^{n=+N}\left(E_{b 0} A_{n}^{y}\right) E_{d} \\
& \psi_{x 0}^{y-\text { top }}=E_{a} k_{z} \sum_{n=-N}^{n=+N}\left(\frac{-E_{c 0}}{\gamma_{n}} C_{n}^{y}\right) E_{d} \\
& \psi_{y 0}^{y-\text { top }}=0 \\
& \psi_{z \text { z } 0}^{y-\text { top }}=E_{a} \sum_{n=-N}^{n=+N}\left(\frac{k_{n}}{\gamma_{n}} E_{c 0} B_{n}^{y}\right) E_{d}
\end{aligned}
$$

\subsection{Solid medium (bottom free surface)}

$$
\begin{aligned}
& \phi_{0}^{y \_ \text {bottom }}=E_{a} \sum_{n=-N}^{n=+N}\left(E_{b 0}^{b} D_{n}^{y}\right) E_{d} \\
& \psi_{x 0}^{y-\text { bottom }}=E_{a} k_{z} \sum_{n=-N}^{n=+N}\left(\frac{-E_{c 0}^{b}}{\gamma_{n}} F_{n}^{y}\right) E_{d} \\
& \psi_{y 0}^{y-\text { bottom }}=0 \\
& \psi_{z 0}^{y}{ }^{\text {bottom }}=E_{a} \sum_{n=-N}^{n=+N}\left(\frac{k_{n}}{\gamma_{n}} E_{c 0}^{b} E_{n}^{y}\right) E_{d}
\end{aligned}
$$

The imposition of the sixth stated boundary conditions for each value of $n$ leads to a system of six equations in the six unknown constants,

$\left[a_{i j}^{y} i=1,6 ; j=1,6\right]\left[c_{i}^{y} i=1,6\right]=\left[b_{i}^{y} i=1,6\right]$

which is fully described in Appendix C.

Once the amplitude of each potential has been calculated, the Green's functions for the displacements are then given by the sum of the source terms and the surface terms originated at the two free slab surfaces,

$$
\begin{aligned}
& G_{x y}^{\mathrm{slab}}=G_{x y}^{\mathrm{full}}+E_{a} \sum_{n=-N}^{n=+N}\left(-i A_{n}^{y} k_{n} E_{b 0}+i B_{n}^{y} k_{n} E_{c 0}\right) E_{d} \\
& +E_{a} \sum_{n=-N}^{n=+N}\left(-i D_{n}^{y} k_{n} E_{b 0}^{b}+i E_{n}^{y} k_{n} E_{c 0}^{b}\right) E_{d} \\
& G_{y y}^{\mathrm{slab}}=G_{y y}^{\mathrm{full}}+E_{a} \sum_{n=-N}^{n=+N}\left[-i v_{n} A_{n}^{y} E_{b 0}+\left(\frac{-i k_{n}^{2}}{\gamma_{n}} B_{n}^{y}+\frac{-i k_{z}^{2}}{\gamma_{n}} C_{n}^{y}\right) E_{c 0}\right] E_{d} \\
& +E_{a} \sum_{n=-N}^{n=+N}\left[-i v_{n} D_{n}^{y} E_{b 0}^{b}+\left(\frac{-i k_{n}^{2}}{\gamma_{n}} E_{n}^{y}+\frac{-i k_{z}^{2}}{\gamma_{n}} F_{n}^{y}\right) E_{c 0}^{b}\right] E_{d}
\end{aligned}
$$

$G_{z y}^{\mathrm{slab}}=G_{z y}^{\mathrm{full}}+E_{a} \sum_{n=-N}^{n=+N}\left(-i A_{n}^{y} k_{z} E_{b 0}\right.$

$\left.+i C_{n}^{y} k_{z} E_{c 0}\right) E_{d}+E_{a} \sum_{n=-N}^{n=+N}\left(-i D_{n}^{y} k_{z} E_{b 0}^{b}\right.$

$\left.+i F_{n}^{y} k_{z} E_{c 0}^{b}\right) E_{d}$ 


\subsection{Load acting in the direction of the z-axis}

Similarly, the discrete form of the displacement potentials, resulting from a spatially sinusoidal harmonic line load along the $z$ direction, applied at the point $\left(x_{0}, y_{0}\right)$ in the $z$ direction, is given by the expressions,

$$
\begin{aligned}
& \phi^{z}=E_{a} k_{z} \sum_{n=-N}^{n=+N}\left(\frac{E_{b}}{v_{n}}\right) E_{d} \\
& \psi_{x}^{z}=E_{a} \sum_{n=-N}^{n=+N}\left(\operatorname{sgn}\left(y-y_{0}\right) E_{c}\right) E_{d} \\
& \psi_{y}^{z}=E_{a} \sum_{n=-N}^{n=+N}\left(\frac{-k_{n}}{\gamma_{n}} E_{c}\right) E_{d} \\
& \psi_{z}^{z}=0
\end{aligned}
$$

The Green's functions for the two-and-a-half dimensional full space are then,

$$
\begin{aligned}
& G_{x z}^{\mathrm{full}}=G_{z x}^{\mathrm{full}}=E_{a} \sum_{n=-N}^{n=+N}\left[\frac{-i k_{z} k_{n}}{v_{n}} E_{b}+\frac{i k_{z} k_{n}}{\gamma_{n}} E_{c}\right] E_{d} G_{y z}^{\mathrm{full}} \\
& =G_{z y}^{\mathrm{full}}=E_{a} \sum_{n=-N}^{n=+N}\left[-i \operatorname{sgn}\left(y-y_{0}\right) k_{z} E_{b}+i \operatorname{sgn}(y\right. \\
& \left.\left.-y_{0}\right) k_{z} E_{c}\right] E_{d} G_{z z}^{\mathrm{full}}=E_{a} \sum_{n=-N}^{n=+N}\left[\frac{-i k_{z}^{2}}{v_{n}} E_{b}+\left(\frac{-i k_{n}^{2}}{\gamma_{n}}\right.\right. \\
& \left.\left.-i \gamma_{n}\right) E_{c}\right] E_{d}
\end{aligned}
$$

The surface terms generated at the two free slab surfaces can be expressed in the form

\subsection{Solid medium (top interface)}

$$
\begin{aligned}
& \phi_{0}^{z-\operatorname{top}}=E_{a} k_{z}^{n=+N}\left(\frac{E_{b 0}}{v_{n}} A_{n}^{z}\right) E_{d} \\
& \psi_{x 0}^{z-\text { top }}=E_{a} \sum_{n=-N}^{n=+N}\left(E_{c 0} B_{n}^{z}\right) E_{d} \\
& \psi_{y 0}^{z-\mathrm{top}}=E_{a} \sum_{n=-N}^{n=+N}\left(\frac{-k_{n}}{\gamma_{n}} E_{c 0} C_{n}^{z}\right) E_{d} \\
& \psi_{z 0}^{z-\mathrm{top}}=0
\end{aligned}
$$

\subsection{Solid medium (bottom interface)}

$$
\begin{aligned}
& \phi_{0}^{z \text { bottom }}=E_{a} k_{z} \sum_{n=-N}^{n=+N}\left(\frac{E_{b 0}^{b}}{v_{n}} D_{n}^{z}\right) E_{d} \\
& \psi_{x 0}^{z-\text { bottom }}=E_{a} \sum_{n=-N}^{n=+N}\left(E_{c 0}^{b} E_{n}^{z}\right) E_{d}
\end{aligned}
$$

$$
\begin{aligned}
& \psi_{y 0}^{z \text { bottom }}=E_{a} \sum_{n=-N}^{n=+N}\left(\frac{-k_{n}}{\gamma_{n}} E_{c 0}^{b} F_{n}^{z}\right) E_{d} \\
& \psi_{z=0}^{z-\text { bottom }}=0
\end{aligned}
$$

The imposition of the sixth stated boundary conditions for each value of $n$ leads to a system of six equations in the six unknown constants,

$\left[a_{i j}^{z} i=1,6 ; j=1,6\right]\left[c_{i}^{z} i=1,6\right]=\left[b_{i}^{z} i=1,6\right]$

and this is fully described in Appendix D.

Once the unknown amplitude of each potential has been calculated, the Green's functions are expressed by the sum of the source terms and the surface terms originated on both free slab surfaces, leading to the following expressions,

$$
\begin{aligned}
& G_{x z}^{\mathrm{slab}}=G_{x z}^{\mathrm{full}}+E_{a} \sum_{n=-N}^{n=+N}\left(\frac{-i k_{z} k_{n}}{v_{n}} A_{n}^{z} E_{b 0}+\frac{i k_{z} k_{n}}{\gamma_{n}} C_{n}^{z} E_{c 0}\right) E_{d} \\
& +E_{a} \sum_{n=-N}^{n=+N}\left(\frac{-i k_{z} k_{n}}{v_{n}} D_{n}^{z} E_{b 0}^{b}+\frac{i k_{z} k_{n}}{\gamma_{n}} F_{n}^{z} E_{c 0}^{b}\right) E_{d} \\
& G_{y z}^{\mathrm{slab}}=G_{y z}^{\mathrm{full}}+E_{a} \sum_{n=-N}^{n=+N}\left(-i k_{z} A_{n}^{z} E_{b 0}+i B_{n}^{z} k_{z} E_{c 0}\right) E_{d} \\
& +E_{a} \sum_{n=-N}^{n=+N}\left(i k_{z} D_{n}^{z} E_{b 0}^{b}+i E_{n}^{z} k_{z} E_{c 0}^{b}\right) E_{d} \\
& G_{z z}^{\mathrm{slab}}=G_{z z}^{\mathrm{full}}+E_{a} \sum_{n=-N}^{n=+N}\left[\frac{-i k_{z}^{2}}{v_{n}} A_{n}^{z} E_{b 0}+\left(\frac{-i k_{n}^{2}}{\gamma_{n}} C_{n}^{z}-i \gamma_{n} B_{n}^{z}\right) E_{c 0}\right] E_{d} \\
& +E_{a} \sum_{n=-N}^{n=+N}\left[\frac{-i k_{z}^{2}}{v_{n}} D_{n}^{z} E_{b 0}^{b}+\left(\frac{-i k_{n}^{2}}{\gamma_{n}} F_{n}^{z}-i \gamma_{n} E_{n}^{z}\right) E_{c 0}^{b}\right] E_{d}
\end{aligned}
$$

Notice that, if $k_{z}=0$ is used, the system of equations derived above is reduced to four unknowns, leading to the two-dimensional Green's function for plane strain line-loads.

\section{Validation of the solution}

The analytical expressions described above were used to calculate the three displacement fields generated by a spatially harmonic varying line load in the $z$ direction in a slab $10.0 \mathrm{~m}$ thick. The results provided were then compared with those arrived at by using the BEM. The BEM code uses a direct formulation in the frequency domain and applies the Green's functions for a full space. The discretization of both slab surfaces is thus required.

An unlimited discretization of the free surface can be avoided by using complex frequencies with a small imaginary part, of the form $\omega_{c}=\omega-i \eta$ [with $\eta=0.7(2 \pi / T)][10,11]$. Boundary elements make a significant contribution to the response for a certain value 


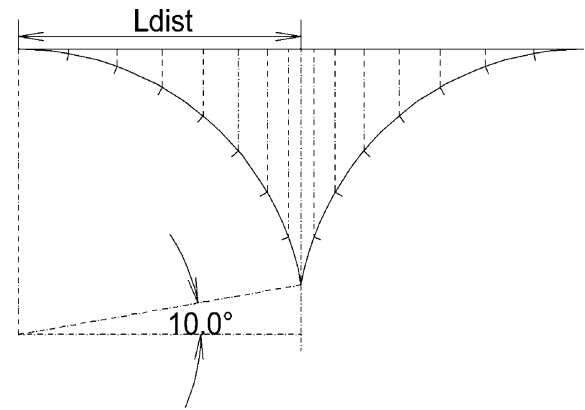

Fig. 2. Definition of the boundary elements.

of damping, but are otherwise unnecessary. These elements are distributed along the surface up to a spatial distance $\left(L_{\text {dist }}\right)$ from the center, given by $L_{\text {dist }}=\alpha T$.

The scheme adopted here for determining the placement and size of the boundary elements uses a geometrical construction, by which an auxiliary circular arc is divided into equal segments according to a previously defined ratio between the wavelength of the dilatational waves and the length of boundary elements. The boundary elements are then defined on the two free surfaces by the vertical projection of these segments. The radius of the required circular arc $(R)$ is greater than $\left(2 L_{\mathrm{dist}}\right) / 2$ and is placed at a tangent to the topographic surface at its boundary discretization end, thus making excessively small boundary elements unnecessary. In this work $R$ is assumed to be $\left[\left(2 L_{\text {dist }}\right) / 2\right] / \cos 10^{\circ}$ (see Fig. 2$)$.
A harmonic point source was applied to the slab medium ( $\alpha=4208 \mathrm{~m} / \mathrm{s}, \beta=2656 \mathrm{~m} / \mathrm{s}$ with $\left.\rho=2140 \mathrm{~kg} / \mathrm{m}^{3}\right)$, at the source point $(x=1.0 \mathrm{~m}, y=2.0 \mathrm{~m})$, acting along the directions $x, y$ and $z$ independently. Calculations are performed in the frequency range $[2.50,320.0 \mathrm{~Hz}]$ with a frequency increment of $2.5 \mathrm{~Hz}$. The scattered displacement field $G_{i j}^{\text {surf }}$ (surface terms), (the displacement in the $i$ direction due to a load acting along $j$ ), is calculated at a receiver point placed at $x=3.0 \mathrm{~m}$ and $y=5.0 \mathrm{~m}$. The imaginary part of the frequency has been set to $\eta=0.7(2 \pi / T)$ with $T=0.0466 \mathrm{~s}$.

The results are calculated for a single value of $k_{z}\left(k_{z}=0.4 \mathrm{rad} / \mathrm{m}\right)$, to prove that the analytical expressions are correct. The real and imaginary parts of the displacements are shown in Figs. 3-5, the analytical responses are represented by the solid lines, while the marked points correspond to the BEM solution. The square and round marks indicate the real and imaginary part of the responses, respectively. The BEM solution required the use of a very large number of boundary elements, defined by the ratio between the wavelength of the incident waves and the length of the boundary elements, which was kept to a minimum of 80 .

It is obvious that these two solutions are in very close agreement, and equally good results were obtained from tests in which loads and receivers were situated at different points.

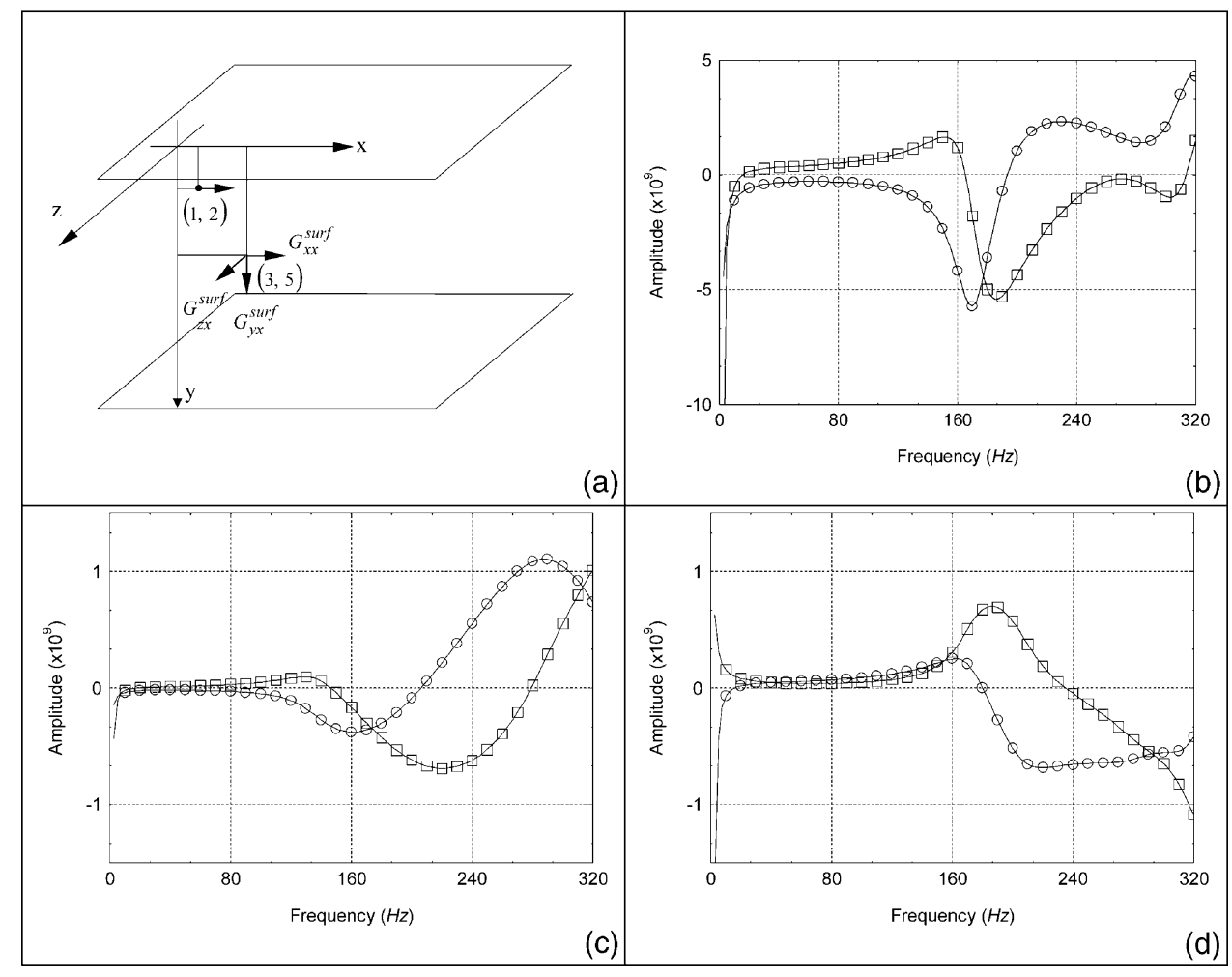

Fig. 3. Spatially sinusoidal harmonic line load along the $z$ direction in a slab, applied in the $x$ direction: (a) Geometry of the problem; (b) $G_{x x}^{\text {surf }}$ solutions; (c) $G_{y x}^{\text {surf }}$ solutions; (d) $G_{z x}^{\text {surf }}$ solutions. 


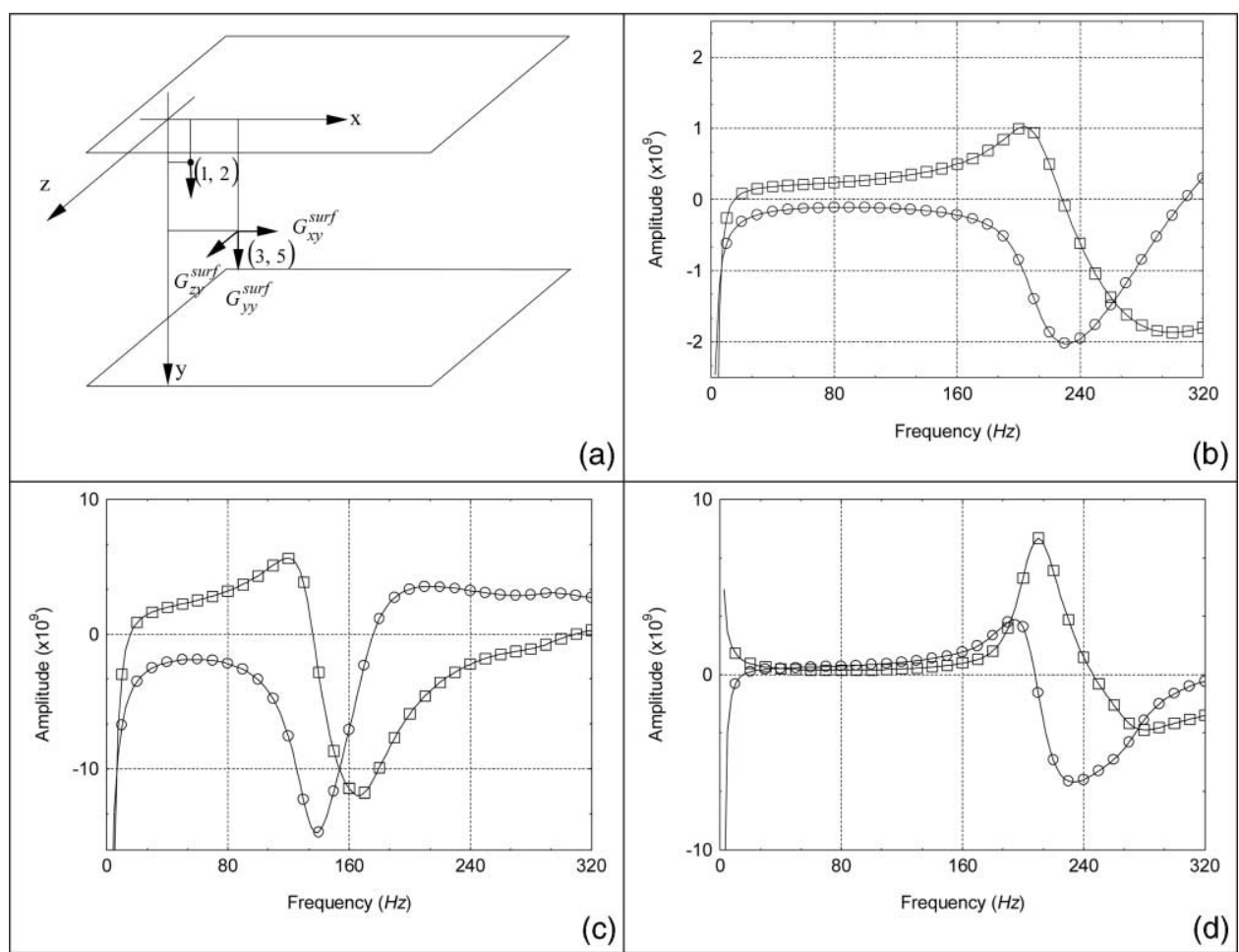

Fig. 4. Spatially sinusoidal harmonic line load along the $z$ direction in a slab, applied in the $y$ direction: (a) Geometry of the problem; (b) $G_{x y}^{\text {surf }}$ solutions; (c) $G_{y y}^{\text {surf }}$ solutions; (d) $G_{z y}^{\text {surf }}$ solutions.

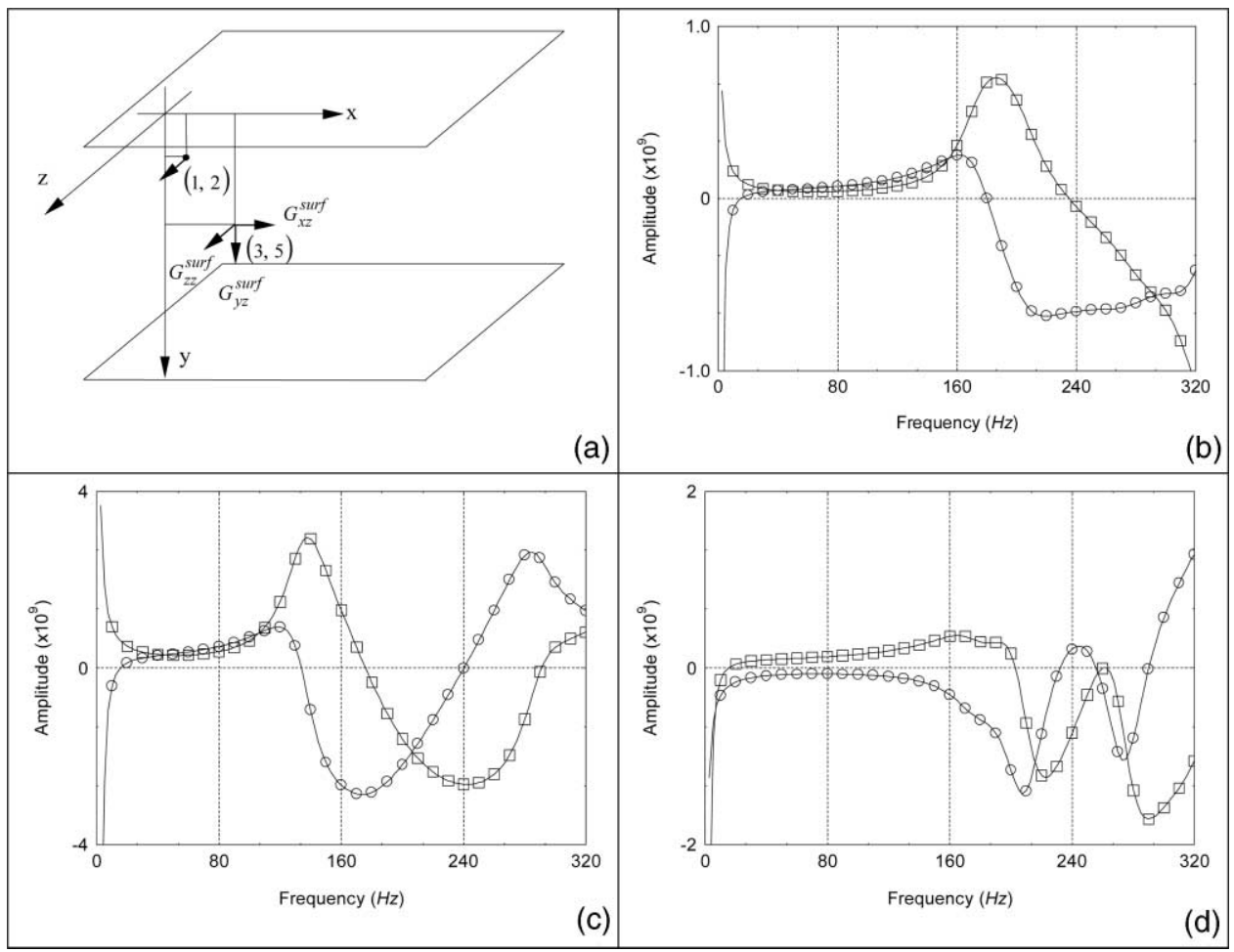

Fig. 5. Spatially sinusoidal harmonic line load along the $z$ direction in a slab, applied in the $z$ direction: (a) Geometry of the problem; (b) $G_{x z}^{\text {surf }}$ solutions; (c) $G_{y z}^{\text {surf }}$ solutions; (d) $G_{z z}^{\text {surf }}$ solutions. 


\section{Conclusions}

Having successfully obtained a wholly analytical solution for the steady state response of a spatially sinusoidal, harmonic line load in a homogeneous three-dimensional slab, we compared the final expressions with the numerical results calculated with the BEM, in order to validate them. The solutions were found to be in very close agreement when the free surfaces were both discretized with a large number of boundary elements.

Appendix A. The Green's function for a two-anda-half dimensional full-space

$$
\begin{aligned}
G_{x x}^{\text {full }} & =\frac{i}{4 \rho \omega^{2}}\left[k_{s}^{2} H_{0 \beta}-\frac{1}{r} B_{1}+\left(\frac{x-x_{0}}{r}\right)^{2} B_{2}\right] \\
G_{y y}^{\text {full }} & =\frac{i}{4 \rho \omega^{2}}\left[k_{s}^{2} H_{0 \beta}-\frac{1}{r} B_{1}+\left(\frac{y-y_{0}}{r}\right)^{2} B_{2}\right] \\
G_{z z}^{\text {full }} & =\frac{i}{4 \rho \omega^{2}}\left[k_{s}^{2} H_{0 \beta}-k_{z}^{2} B_{0}\right] \\
G_{x y}^{\text {full }} & =G_{y x}^{\text {full }}=\frac{i}{4 \rho \omega^{2}}\left(\frac{x-x_{0}}{r}\right)\left(\frac{y-y_{0}}{r}\right) B_{2} \\
G_{x z}^{\text {full }} & =G_{z x}^{\text {full }}=\frac{-k_{z}}{4 \rho \omega^{2}}\left(\frac{x-x_{0}}{r}\right) B_{1} \\
G_{y z}^{\text {full }} & =G_{z y}^{\text {full }}=\frac{-k_{z}}{4 \rho \omega^{2}}\left(\frac{y-y_{0}}{r}\right) B_{1}
\end{aligned}
$$

with

$$
\begin{aligned}
& r=\sqrt{\left(x-x_{0}\right)^{2}+\left(y-y_{0}\right)^{2}} \\
& H_{n \alpha}=H_{n}^{(2)}\left(k_{\alpha} r\right) \\
& H_{n \beta}=H_{n}^{(2)}\left(k_{\beta} r\right) \\
& B_{n}=k_{\beta}^{n} H_{n \beta}-k_{\alpha}^{n} H_{n \alpha}
\end{aligned}
$$

Appendix B. Definition of $\left(a_{i j}^{x} i=1,6 ; j=1,6\right),\left(c_{i}^{x}\right.$ $i=1,6)$ and $\left(b_{i}^{x} i=1,6\right)$

$$
\begin{array}{lll}
a_{i j}^{x} i=1,6 ; j=1,6 & & \\
a_{11}^{x}=-2 k_{n}^{2} & a_{12}^{x}=-k_{z}^{2} & a_{13}^{x}=k_{n}^{2}-\gamma_{n}^{2} \\
a_{14}^{x}=-a_{11}^{x} E_{b h} & a_{15}^{x}=-a_{12}^{x} E_{c h} & a_{16}^{x}=-a_{13}^{x} E_{c h} \\
a_{21}^{x}=-2 & a_{22}^{x}=1 & a_{23}^{x}=1 \\
a_{24}^{x}=-a_{21}^{x} E_{b h} & a_{25}^{x}=-a_{22}^{x} E_{c h} & a_{26}^{x}=-a_{23}^{x} E_{c h} \\
a_{31}^{x}=\frac{-k_{z}^{2}}{v_{n}}-\frac{2 v_{z n}^{2}}{v_{n}} & a_{32}^{x}=0 & a_{33}^{x}=2 \gamma_{n} \\
a_{34}^{n}=a_{31}^{x} E_{b h} & a_{35}^{x}=0 & a_{36}^{x}=a_{33}^{x} E_{c h} \\
a_{41}^{x}=a_{11}^{x} E_{b h} & a_{42}^{x}=a_{12}^{x} E_{c h} & a_{43}^{x}=a_{13}^{x} E_{c h} \\
a_{44}^{x}=-a_{11}^{x} & a_{45}=-a_{12}^{x} & a_{46}^{x}=-a_{13}^{x} \\
a_{51}^{x}=a_{21}^{x} E_{b h} & a_{52}^{x}=a_{22}^{x} E_{c h} & a_{53}^{x}=a_{23}^{x} E_{c h} \\
a_{54}^{x}=-a_{21}^{x} & a_{55}^{x}=-a_{22}^{x} & a_{56}^{x}=-a_{23}^{x} \\
a_{61}^{x}=a_{31}^{x} E_{b h} & a_{62}^{x}=0 & a_{63}^{x}=a_{33}^{x} E_{c h} \\
a_{64}^{x}=a_{31}^{x} & a_{65}^{x}=0 & a_{66}^{x}=a_{33}^{x}
\end{array}
$$

$$
\begin{array}{rlrl}
c_{1}^{x} i & =1,6 & & \\
c_{1}^{x} & =A_{n}^{x} & c_{2}^{x}=B_{n}^{x} & c_{3}^{x}=C_{n}^{x} \\
c_{4}^{x}=D_{n}^{x} & c_{5}^{x}=E_{n}^{x} & c_{6}^{x}=F_{n}^{x}
\end{array}
$$

$b_{i}^{x} i=1,6$

$b_{1}^{x}=-2 k_{n}^{2} E_{b 1}+\left(-k_{s}^{2}+2 k_{n}^{2}\right) E_{c 1} \quad b_{2}^{x}=-2 E_{b 1}+2 E_{c 1}$

$b_{3}^{x}=\left(\frac{k_{s}^{2}}{v_{n}}+\frac{2 v_{z n}^{2}}{v_{n}}\right) E_{b 1}-2 \gamma_{n} E_{c 1} \quad b_{4}^{x}=2 k_{n}^{2} E_{b h 1}-\left(-k_{s}^{2}+2 k_{n}^{2}\right) E_{c h 1}$

$$
b_{5}^{x}=2 E_{b h 1}-2 E_{c h 1} \quad b_{6}^{x}=\left(\frac{k_{s}^{2}}{v_{n}}+\frac{2 v_{z n}^{2}}{v_{n}}\right) E_{b h 1}-2 \gamma_{n} E_{c h 1}
$$

with $\quad E_{b 1}=\mathrm{e}^{-i v_{n} y_{0}}, \quad E_{c 1}=\mathrm{e}^{-i \gamma_{n} y_{0}}, \quad v_{z n}=\sqrt{-k_{z}^{2}-k_{n}^{2}}, \quad E_{b h}=\mathrm{e}^{-i v_{n} h}$, $E_{c h}=\mathrm{e}^{-i \gamma_{n} h}, E_{b h 1}=\mathrm{e}^{-i v_{n}\left|h-y_{0}\right|}$ and $E_{c h 1}=\mathrm{e}^{-i \gamma_{n}\left|h-y_{0}\right|}$.

\section{Appendix C. Definition of $\left(a_{i j}^{y} i=1,6 ; j=1,6\right),\left(c_{i}^{y}\right.$} $i=1,6)$ and $\left(b_{i}^{y} i=1,6\right)$

$a_{i j}^{y} i=1,6 ; j=1,6$

$$
\begin{aligned}
& a_{11}^{y}=-2 v_{n} \quad a_{12}^{y}=\frac{-k_{n}^{2}}{\gamma_{n}}+\gamma_{n} \quad a_{13}^{y}=\frac{-k_{z}^{2}}{\gamma_{n}} \\
& a_{14}^{y}=a_{11}^{y} E_{b h} \quad a_{15}^{y}=a_{12}^{y} E_{c h} \quad a_{16}^{y}=a_{13}^{y} E_{c h} \\
& a_{21}^{y}=-2 v_{n} \quad a_{22}^{y}=\frac{-k_{n}^{2}}{\gamma_{n}} \quad a_{23}^{y}=\frac{-k_{z}^{2}}{\gamma_{n}}+\gamma_{n} \\
& a_{24}^{y}=a_{21}^{y} E_{b h} \quad a_{25}^{y}=a_{22}^{y} E_{c h} \quad a_{26}^{y}=a_{23}^{y} E_{c h} \\
& a_{31}^{y}=-k_{s}^{2}-2 v_{z n}^{2} \quad a_{32}^{y}=-2 k_{n}^{2} \quad a_{33}^{y}=-2 k_{z}^{2} \\
& a_{34}^{y}=-a_{31}^{y} E_{b h} \quad a_{35}^{y}=-a_{32}^{y} E_{c h} \quad a_{36}^{y}=-a_{33}^{y} E_{c h} \\
& a_{41}^{y}=a_{11}^{y} E_{b h} \quad a_{42}^{y}=a_{12}^{y} E_{c h} \quad a_{43}^{y}=a_{13}^{y} E_{c h} \\
& a_{44}^{y}=a_{11}^{y} \quad a_{45}^{y}=a_{12}^{y} \quad a_{46}^{y}=a_{13}^{y} \\
& a_{51}^{y}=a_{21}^{y} E_{b h} \quad a_{52}^{y}=a_{22}^{y} E_{c h} \quad a_{53}^{y}=a_{23}^{y} E_{c h} \\
& a_{54}^{y}=a_{21}^{y} \quad a_{55}^{y}=a_{22}^{y} \quad a_{56}^{y}=a_{23}^{y} \\
& a_{61}^{y}=a_{31}^{y} E_{b h} \quad a_{62}^{y}=a_{32}^{y} E_{c h} \quad a_{63}^{y}=a_{33}^{y} E_{c h} \\
& a_{64}^{y}=-a_{31}^{y} \quad a_{65}^{y}=-a_{32}^{y} \quad a_{66}^{y}=-a_{33}^{y} \\
& c_{i}^{y} i=1,6 \\
& c_{1}^{y}=A_{n}^{y} \quad c_{2}^{y}=B_{n}^{y} \quad c_{3}^{y}=C_{n}^{y} \\
& c_{4}^{y}=D_{n}^{y} \quad c_{5}^{y}=E_{n}^{y} \quad c_{6}^{y}=F_{n}^{y}
\end{aligned}
$$

$$
\begin{array}{ll}
b_{1}^{y}=2 v_{n} E_{b 1}-\left(\frac{v_{z n}^{2}}{\gamma_{n}}+\gamma_{n}\right) E_{c 1} & b_{2}^{y}=2 v_{n} E_{b 1}-\left(\frac{v_{z n}^{2}}{\gamma_{n}}+\gamma_{n}\right) E_{c 1} \\
b_{3}^{y}=\left(-k_{s}^{2}-2 v_{z n}^{2}\right) E_{b 1}+2 v_{z n}^{2} E_{c 1} & b_{4}^{y}=2 v_{n} E_{b h 1}-\left(\frac{v_{z n}^{2}}{\gamma_{n}}+\gamma_{n}\right) E_{c h 1} \\
b_{5}^{y}=2 v_{n} E_{b h 1}-\left(\frac{v_{z n}^{2}}{\gamma_{n}}+\gamma_{n}\right) E_{c h 1} & b_{6}^{y}=\left(k_{s}^{2}+2 v_{z n}^{2}\right) E_{b h 1}-2 v_{z n}^{2} E_{c h 1}
\end{array}
$$


with $E_{b 1}=\mathrm{e}^{-i v_{n} y_{0}}, \quad E_{c 1}=\mathrm{e}^{-i \gamma_{n} y_{0}}, \quad v_{z n}=\sqrt{-k_{z}^{2}-k_{n}^{2}}, \quad E_{b h}=\mathrm{e}^{-i v_{n} h}$, $E_{c h}=\mathrm{e}^{-i \gamma_{n} h}, E_{b h 1}=\mathrm{e}^{-i v_{n}\left|h-y_{0}\right|}$ and $E_{c h 1}=\mathrm{e}^{-i \gamma_{n}\left|h-y_{0}\right|}$.

Appendix D. Definition of $\left(a_{i j}^{z} i=1,6 ; j=1,6\right),\left(c_{i}^{z}\right.$ $i=1,6)$ and $\left(b_{i}^{z} i=1,6\right)$

$a_{i j}^{z} i=1,6 ; j=1,6$

$a_{11}^{z}=-2$

$a_{12}^{z}=1 \quad a_{13}^{z}=1$

$a_{14}^{z}=-a_{11}^{z} E_{b h}$

$a_{15}^{z}=-a_{12}^{z} E_{c h} \quad a_{16}^{z}=-a_{13}^{z} E_{c h}$

$a_{21}^{z}=-2 k_{z}^{2}$

$a_{22}^{z}=k_{z}^{2}-\gamma_{n}^{2} \quad a_{23}^{z}=-k_{n}^{2}$

$a_{24}^{z}=-a_{21}^{z} E_{b h}$

$a_{25}^{z}=-a_{22}^{z} E_{c h} \quad a_{26}^{z}=-a_{23}^{z} E_{c h}$

$a_{31}^{z}=-\left(\frac{k_{s}^{2}}{v_{n}}+\frac{2 v_{z n}^{2}}{v_{n}}\right)$

$a_{32}^{z}=2 \gamma_{n} \quad a_{33}^{z}=0$

$a_{34}^{z}=a_{31}^{z} E_{b h}$

$a_{35}^{z}=a_{32}^{z} E_{c h} \quad a_{36}^{z}=0$

(D1)

$a_{41}^{z}=a_{11}^{z} E_{b h}$

$a_{42}^{Z}=a_{12}^{z} E_{c h} \quad a_{43}^{z}=a_{13}^{z} E_{c h}$

$a_{44}^{z}=-a_{11}^{z}$

$a_{45}^{z}=-a_{12}^{z}$

$a_{46}^{z}=-a_{13}^{z}$

$a_{51}^{z}=a_{21}^{z} E_{b h}$

$a_{52}^{z}=a_{22}^{z} E_{c h}$

$a_{53}^{z}=a_{23}^{z} E_{c h}$

$a_{54}^{z}=-a_{21}^{z}$

$a_{55}^{z}=-a_{22}^{z}$

$a_{56}^{z}=-a_{23}^{z}$

$a_{61}^{z}=a_{31}^{z} E_{b h}$

$a_{62}^{z}=a_{32}^{z} E_{c h} \quad a_{63}^{z}=0$

$a_{64}^{z}=a_{31}^{z}$

$a_{65}^{z}=a_{32}^{z} \quad a_{66}^{z}=0$

$c_{i}^{z} i=1,6$

$c_{1}^{z}=A_{n}^{z} \quad c_{2}^{z}=B_{n}^{z} \quad c_{3}^{z}=C_{n}^{z}$

$c_{4}^{z}=D_{n}^{z} \quad c_{5}^{z}=E_{n}^{z} \quad c_{6}^{z}=F_{n}^{z}$

with $\quad E_{b 1}=\mathrm{e}^{-i v_{n} y_{0}}, \quad E_{c 1}=\mathrm{e}^{-i \gamma_{n} y_{0}}, \quad v_{z n}=\sqrt{-k_{z}^{2}-k_{n}^{2}}, \quad E_{b h}=\mathrm{e}^{-i v_{n} h}$, $E_{c h}=\mathrm{e}^{-i \gamma_{n} h}, E_{b h 1}=\mathrm{e}^{-i v_{n}\left|h-y_{0}\right|}$ and $E_{c h 1}=\mathrm{e}^{-i \gamma_{n}\left|h-y_{0}\right|}$.

\section{References}

[1] Tadeu A, António J, Godinho L. Green's function for two-anda-half dimensional elastodynamic problems in a half-space. Computational Mechanics 2001;27(6):484-91.

[2] Tadeu A, Kausel E. Green's functions for two-and-a-half dimensional elastodynamic problems. Journal of Engineering Mechanics, ASCE 2000;126(10):1093-7.

[3] Lamb H. On the propagation of tremors at the surface of an elastic solid. Phil Trans Roy Soc London 1904;A203:1-42.

[4] Bouchon M. Discrete wave number representation of elastic wave fields in three-space dimensions. Journal of Geophysical Research 1979;84:3609-14.

[5] Kim J, Papageorgiou AS. Discrete wavenumber boundary element method for 3-D scattering problems. Journal of Engineering Mechanics ASCE 1993;119(3):603-24.

[6] Kawase H. Time-domain response of a semicircular canyon for incident SV, P and Rayleigh waves calculated by the discrete wavenumber boundary element method. Bulletin of the Seismological Society of America 1988;78:1415-37.

[7] Gradshteyn and Ryzhik. Tables of integrals, series, and products, Academic Press, 1980

[8] Pedersen HA, Sánchez-Sesma FJ, Campillo M. Three-dimensional scattering by two-dimensional topographies. Bulletin of the Seismological Society of America 1994;84:1169-83.

$b_{i}^{z} i=1,6$

$b_{1}^{z}=2\left(-E_{b 1}+E_{c 1}\right) \quad b_{2}^{z}=-2 k_{z}^{2} E_{b 1}+\left(k_{z}^{2}-\gamma_{n}^{2}-k_{n}^{2}\right) E_{c 1}$

$b_{3}^{z}=\left(\frac{k_{s}^{2}}{v_{n}}+\frac{2 v_{z n}^{2}}{v_{n}}\right) E_{b 1}-2 \gamma_{n} E_{c 1} \quad b_{4}^{z}=-2\left(-E_{b h 1}+E_{c h 1}\right)$

$b_{5}^{z}=2 k_{z}^{2} E_{b h 1}-\left(k_{z}^{2}-\gamma_{n}^{2}-k_{n}^{2}\right) E_{c h 1} \quad b_{6}^{z}=\left(\frac{k_{s}^{2}}{v_{n}}+\frac{2 v_{z n}^{2}}{v_{n}}\right) E_{b h 1}-2 \gamma_{n} E_{c h 1}$

[9] Papageorgiou AS, Pei D. A discrete wavenumber boundary element method for study of 3-D response of 2-D scatterers. Earthquake Engng Struct Dyn 1998;27:619-38.

[10] Bouchon M, Aki K. Discrete wave-number representation of seismic-source wave fields. Bulletin of the Seismological Society of America 1977;67:259-77.

[11] Phinney RA. Theoretical calculation of the spectrum of first arrivals in the layered elastic medium. J Geophysics Res 1965;70:5107-23. 